\title{
Magnetic fields of AGB and post-AGB stars
}

\author{
Wouter Vlemmings ${ }^{1}$ \\ ${ }^{1}$ Chalmers University of Technology, Department of Space, Earth and Environment, \\ Onsala Space Observatory, SE-43992 Onsala, Sweden \\ email: wouter.vlemmings@chalmers.se
}

\begin{abstract}
There is ample evidence for strong magnetic fields in the envelopes of (Post-)Asymptotic Giant Branch (AGB) stars as well as supergiant stars. The origin and role of these fields are still unclear. This paper updates the current status of magnetic field observations around AGB, post-AGB stars and describes their possible role during these stages of evolution. The discovery of magnetically aligned dust around a supergiant star is also highlighted. In our search for the origin of the magnetic fields, recent observations show the signatures of possible magnetic activity and rotation, indicating that the magnetic fields might be intrinsic to the AGB stars.
\end{abstract}

Keywords. magnetic fields, polarization, stars: AGB and post-AGB, supergiants, rotation, spots

\section{Introduction}

Magnetic fields are ubiquitous throughout the Universe and play an important role across a wide range of scales. Primordial magnetic fields could have played a role in the formation of the first stars just as magnetic fields in molecular clouds are an important ingredient in current star formation. Magnetic fields have also been detected in almost all stellar types and in almost all phases of stellar evolution (e.g. Berdyugina 2009), and have significant effects on stellar evolution through, e.g. their influence on the internal mixing. The magnetic field of stars can have either a dynamo origin, i.e. be generated by a dynamo process in the star itself (e.g. Charbonneau 2014), or can be the result of a remnant 'fossil' field, which are fields that originate from the star formation process (e.g. Braithwaite \& Spruit 2004). The stellar magnetic field is affected by the changes of physical properties during stellar evolution and, because of flux conservation, becomes increasingly difficult to observe at the stellar surface when the star expands in the final phases of its life. However, in stellar end products, such as white dwarfs and neutron stars, magnetic fields are also shown to be significant.

The role of magnetic fields around AGB stars is not clear. In principle, they could help levitate material off the stellar surface through Alfv'en waves (e.g. Falceta-Gonçalves \& Jatenco-Pereira 2002), or through the creation of cool spots on the surface above with dust can form easier (Soker 1998). A specific model for the AGB star o Ceti (Mira A) has shown that a hybrid magnetohydrodynamic-dust-driven wind scenario can explain its mass loss (Thirumalai \& Heyl 2013). In such a model, Alfvén waves add energy to lift material before dust forms and radiation pressure accelerates a wind. Magnetic fields also play an important role in the internal mixing required for s-process (slow) neutron capture reactions that define the stellar yields (e.g. Trippella et al. 2016).

After the AGB phase, the stellar envelopes undergo a major modification as they evolve to Planetary Nebulae (PNe). The standard assumption is that the initial slow AGB mass loss quickly changes into a fast superwind, generating shocks and accelerating the surrounding envelope (Kwok et al. 1978). It is during this phase that the typically spherical CSE evolves into a Planetary Nebula. As the majority of of pre-PNe are aspherical, an 


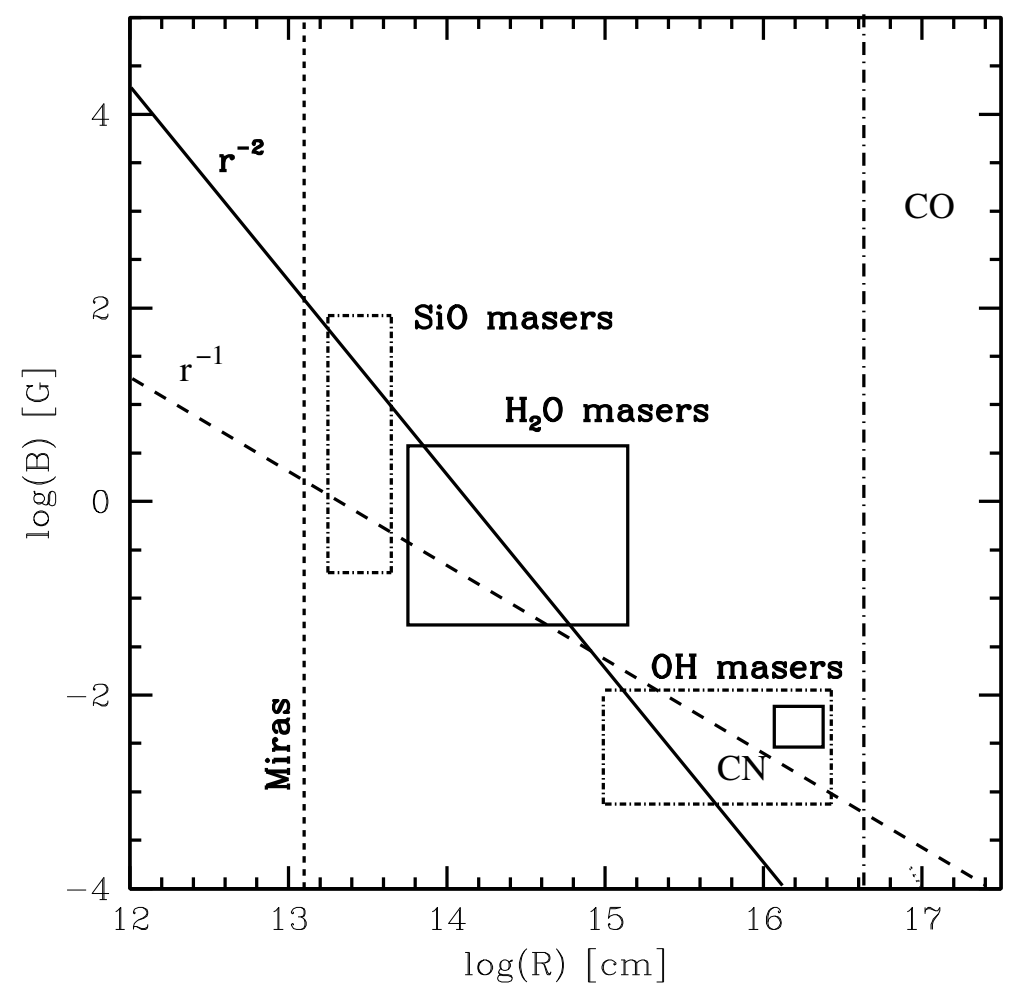

Figure 1. The circumstellar magnetic field strength vs. radius relation as indicated by current (maser) polarization observation. The boxes show the range of observed magnetic field strengths derived from the observations of $\mathrm{SiO}$ masers (Kemball et al. 2009, Herpin et al. 2006), $\mathrm{H}_{2} \mathrm{O}$ masers (Vlemmings et al. 2002,Vlemmings et al. 2005, Leal-Ferreira et al. 2013), OH masers (Rudnitski et al. 2010, Gonidakis et al. 2014) and CN (Duthu et al. 2017). The thick solid and dashed lines indicate an $r^{-2}$ solar-type and $r^{-1}$ toroidal magnetic field configuration. The vertical dashed line indicates the stellar surface. Observations of the Goldreich-Kylafis effect in CO (e.g. Vlemmings et al. 2012) will uniquely probe the outer edge of the envelope (vertical dashed dotted line).

additional mechanism is needed to explain the departure from sphericity. This mechanism is still a matter of fierce debate. One possibility is that the interaction of the post-AGB star and a binary companion or massive planet supports a strong magnetic field that is capable of shaping the outflow (e.g. Nordhaus et al. 2007).

This paper expands on (and partly reproduces) the reviews presented in Vlemmings 2018 and Vlemmings 2014 and I refer interested readers to those review (and references therein) for further background.

\section{Overview of magnetic field observations}

\subsection{AGB stars}

Generally, AGB magnetic field measurements come from maser polarization observations ( $\mathrm{SiO}, \mathrm{H}_{2} \mathrm{O}$ and $\left.\mathrm{OH}\right)$. These have revealed a strong magnetic field throughout the circumstellar envelope. Figure 1, the magnetic field strength in the regions of the envelope traced by the maser measurements throughout AGB envelopes. The field appears to vary between $B \propto R^{-2}$ (solar-type) and $B \propto R^{-1}$ (toroidal). Although the maser observations trace only oxygen-rich AGB stars, recent CN Zeeman splitting observations (Duthu et 


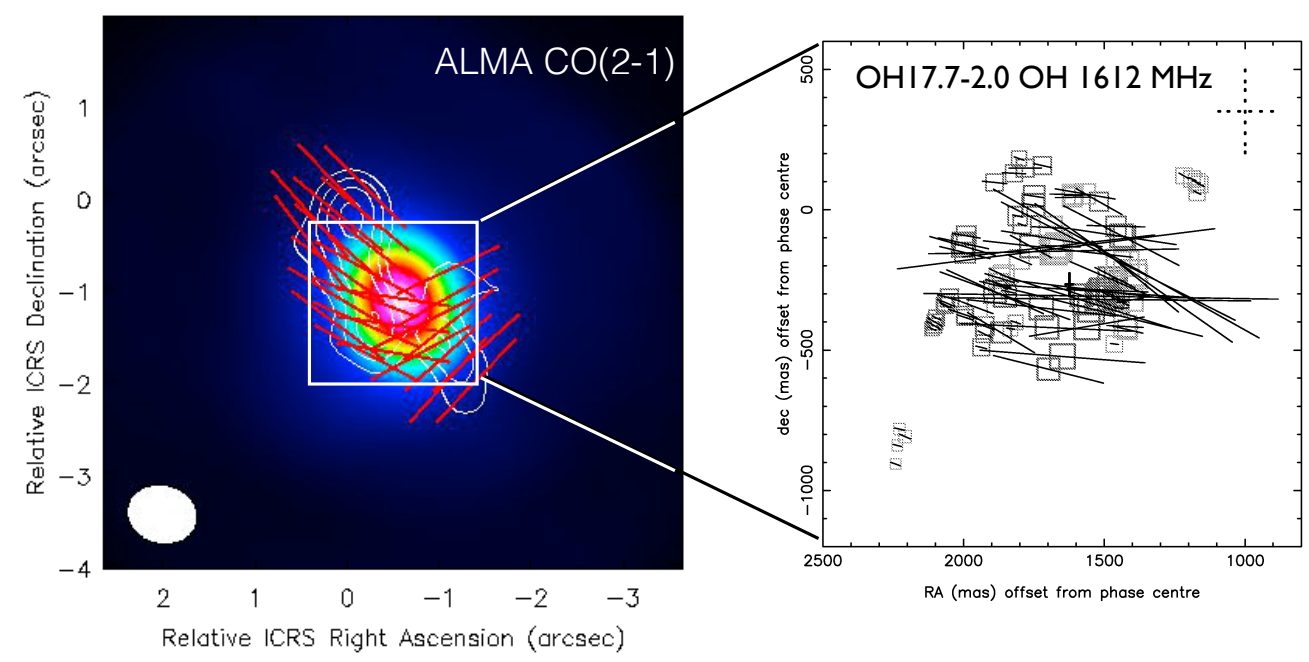

Figure 2. A comparison of the magnetic field determined using ALMA observations of the Goldreich-Kylafis effect on circumstellar CO (left, Tafoya \& Vlemmings, in prep.) and MERLIN observations of $\mathrm{OH}$ masers (right, Bains et al. 2003) around the post-AGB star OH 17.7-2.0. These observations show that the $\mathrm{CO}$ and $\mathrm{OH}$ trace the same large-scale magnetic field.

al. 2017) indicate that similar strength fields are found around carbon-rich stars. The envelope magnetic fields are also consistent with thus far the only direct measurement of the Zeeman effect on the surface of an AGB star, the Mira variable star $\chi$ Cyg (Lèbre et al. 2014). In Table. 1 an overview is given of the energy densities throughout the AGB envelopes.

The large-scale structure of the magnetic field is more difficult to infer, predominantly because the maser observations often probe only limited line-of-sights. Even though specifically $\mathrm{OH}$ observations seem to indicate a systematic field structure, it has often been suggested that there might not be a large-scale component to the field that would be necessary to shape the outflow (Soker 2002). Until recently the only tight shape constraints throughout the envelope had been determined for the field around the supergiant star VX Sgr, where maser observations spanning 3 orders of magnitude in distance are all consistent with a large scale, possibly dipole shaped, magnetic field (Vlemmings et al. 2005, Vlemmings et al. 2011).

Very recent ALMA observations have shown that it will soon be possible to finally overcome the problems with determining the circumstellar magnetic field structure. This involves observations aimed at measuring the Goldreich-Kylafis effect, which allows us to use the polarisation of non-maser molecular lines (in this case $\mathrm{CO}$ ) to determine the magnetic field morphology in the more diffuse circumstellar gas. The first of these observations, for the post-AGB star $\mathrm{OH}$ 17.7-2.0, indicate that the magnetic field structure probed by the $\mathrm{CO}$ is consistent with that derived from $\mathrm{OH}$ maser observations (Fig. 2 Tafoya \& Vlemmings in prep.). This puts to rest the decades old question if maser magnetic field measurements can really be used to probe the large-scale fields. The second set of observations has given us the first view velocity resolved view of the large-scale magnetic field in the AGB stars IRC+10216 (Fig. 3, Vlemmings et al. in prep.). 

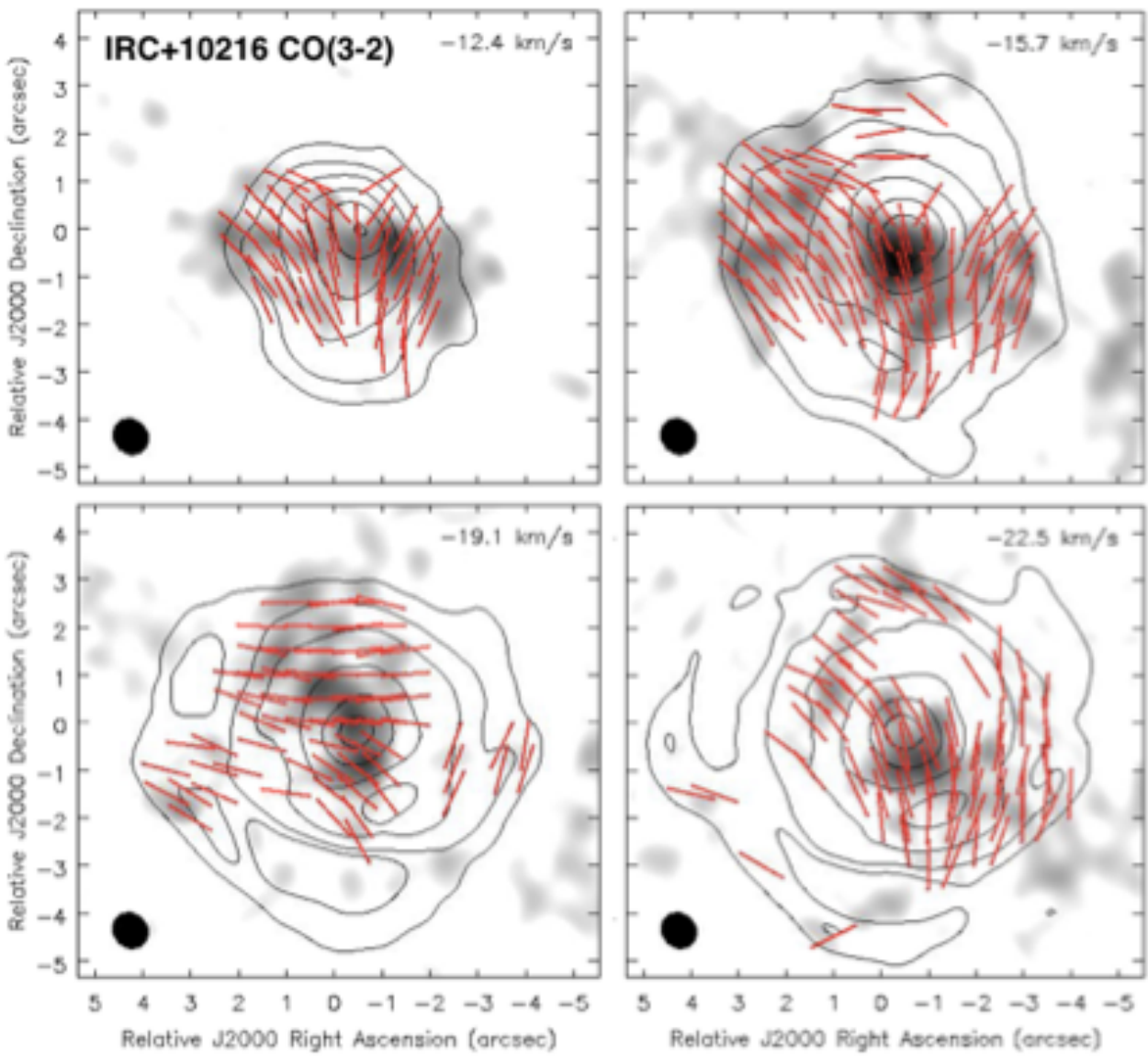

Figure 3. Four channel maps showing the Goldreich-Kylafis effect on the $\mathrm{CO}(3-2)$ line in the envelope of the AGB star IRC+10216. The ALMA observations for the first time clearly resolve the magnetic field structure throughout the CSE. The red vectors indicate the polarisation direction. The linearly polarised emission is shown in greyscale. The contours indicate the total intensity emission. A structure function analysis will be able to reveal the velocity resolved field strength and initial indication indicate a structured field with a strength $>1 \mathrm{G}$ at the stellar surface(Vlemmings et al., in prep.).

\section{2. post-AGB stars}

Similar to the AGB stars, masers are the main source of magnetic field information of post-AGB and P-PNe and even for some $\mathrm{PNe}$. $\mathrm{OH}$ maser observations indicate magnetic field strengths similar to those of AGB stars (few mG) and a clear large scale magnetic field structure (Bains et al. 2003, Gómez et al. 2016). Also dust polarization observations indicate a large scale magnetic field (e.g. Sabin et al. 2015).

Magnetic fields have also been detected around the so-called 'water-fountain' sources. These sources exhibit fast and highly collimated $\mathrm{H}_{2} \mathrm{O}$ maser jets that often extend beyond even the regular $\mathrm{OH}$ maser shell. With the dynamical age of the jet of order 100 years, they potentially are the progenitors of the bipolar (P-)PNe. Observations of the arch-type of the water-fountains, W43A, have revealed a strong toroidal magnetic field that is collimating the jet (Vlemmings et al. 2006). For another water-fountain source, IRAS 15445-5449, a synchrotron jet related to strong magnetic fields has been detected 
Table 1. Energy densities in AGB envelopes

\begin{tabular}{|c|c|c|c|c|c|c|}
\hline & & Photosphere & $\mathrm{SiO}$ & $\mathrm{H}_{2} \mathrm{O}$ & $\mathrm{OH}$ & $\mathrm{CO} / \mathrm{CN}$ \\
\hline $\begin{array}{l}B \\
R \\
V_{\exp } \\
n_{\mathrm{H}_{2}} \\
T\end{array}$ & $\begin{array}{l}{[\mathrm{G}]} \\
{[\mathrm{AU}]} \\
{\left[\mathrm{km} \mathrm{s}^{-1}\right]} \\
{\left[\mathrm{cm}^{-3}\right]} \\
{[\mathrm{K}]}\end{array}$ & $\begin{array}{c}\sim 1-10 ? \\
\quad- \\
\sim 20 \\
\sim 10^{11} \\
\sim 2500\end{array}$ & $\begin{aligned} & \sim 3.5 \\
& \sim 3 \\
& \sim 5 \\
\sim & 10^{10} \\
\sim & 1300\end{aligned}$ & $\begin{array}{l}\sim 0.3 \\
\sim 25 \\
\sim 8 \\
\sim 10^{8} \\
\sim 500\end{array}$ & $\begin{aligned} \sim & 0.003 \\
& \sim 50 \\
& \sim 10 \\
& \sim 10^{6} \\
\sim & 300\end{aligned}$ & $\begin{array}{c}\sim 0.003-0.008 \\
\sim 50-100 \\
\sim 10 \\
\sim 10^{5} \\
\sim 150\end{array}$ \\
\hline $\begin{array}{l}B^{2} / 8 \pi \\
n K T \\
\rho V_{\exp }^{2} \\
V_{A}\end{array}$ & $\begin{array}{l}{\left[\text { dyne } \mathrm{cm}^{-2}\right]} \\
{\left[\text { dyne } \mathrm{cm}^{-2}\right]} \\
{\left[\text { dyne } \mathrm{cm}^{-2}\right]} \\
{\left[\mathrm{km} \mathrm{s}^{-1}\right]}\end{array}$ & $\begin{array}{c}\mathbf{1 0}^{-\mathbf{1 . 4}+\mathbf{0 . 6}} \\
10^{-1.5} \\
10^{-0.3} \\
\sim 20\end{array}$ & $\begin{array}{l}\mathbf{1 0}^{+\mathbf{0 . 1}} \\
10^{-2.7} \\
10^{-2.5} \\
\sim 100\end{array}$ & $\begin{array}{l}\mathbf{1 0}^{-\mathbf{2 . 4}} \\
10^{-5.2} \\
10^{-4.1} \\
\sim 300\end{array}$ & $\begin{array}{l}10^{-6.4} \\
10^{-7.4} \\
\mathbf{1 0}^{-\mathbf{5 . 9}} \\
\sim 8\end{array}$ & $\begin{array}{c}\mathbf{1 0}^{-\mathbf{6 . 0},-6.4} \\
10^{-8.7} \\
10^{-6.9} \\
\sim 8\end{array}$ \\
\hline
\end{tabular}

Table 2. Energy densities through AGB star envelopes. From left to right the columns indicate the stellar photosphere, maser regions and the region probed by $\mathrm{CO} / \mathrm{CN}$, with increasing distance to the central star. The top rows are the typical magnetic field strength $B$, distance to the star $R$, expansion velocity $V_{\exp }$, hydrogen number density $n_{\mathrm{H}}$ and temperature $T$. The bottom rows are the magnetic, thermal and kinematic energy and a rough estimate of the Alvfén velocity $V_{A}$.

(Pérez-Sánchez et al. 2013). Similar, synchrotron emission has been found from what could be one of the youngest PNe (Suárez et al. 2015).

Finally, recently also surface fields have been measured for 2 post-AGB stars (Sabin et al. 2015). These fields are consistent with the fields inferred from the envelope measurements

\subsection{Supergiant stars}

Many maser observations show that strong magnetic fields are also present in the envelopes of Red Supergiant stars (e.g. Vlemmings et al. 2002, Herpin et al. 2006). The questions about local or large scale fields, are the same as around AGB stars. As noted above, the supergiant VX Sgr is one of the first stars where a large scale magnetic field, with a structure consistent throughout the envelope, was found. At (sub-)millimeter wavelengths it is now possible to simultaneously study the polarization of masers, regular molecular lines, and circumstellar dust using ALMA. Recent observations of VY CMa indicate magnetically aligned dust and consistent structures between the maser and nonmaser molecular lines (Fig. 4. Vlemmings et al. 2017). The observations indicate that magnetic fields could be involved in the mass loss of these massive stars.

\section{Indirect tracers and origin of the magnetic field}

The origin of AGB magnetic fields is unclear and might require an extra source of angular momentum to maintain a stellar dynamo. This however depends strongly on the magnetic coupling throughout the star itself. If a sufficiently strong magnetic field persist at the AGB stellar surface, it might be possible to detect signs of magnetic activity. Recently, it has been shown that the majority of the AGB stars are UV-emitters (Montez et al. 2017) which could be a sign of (magnetic) activity. Similarly, recent observation of the surface of the AGB star W Hya show high brightness temperature hotspots (Fig 5 . 

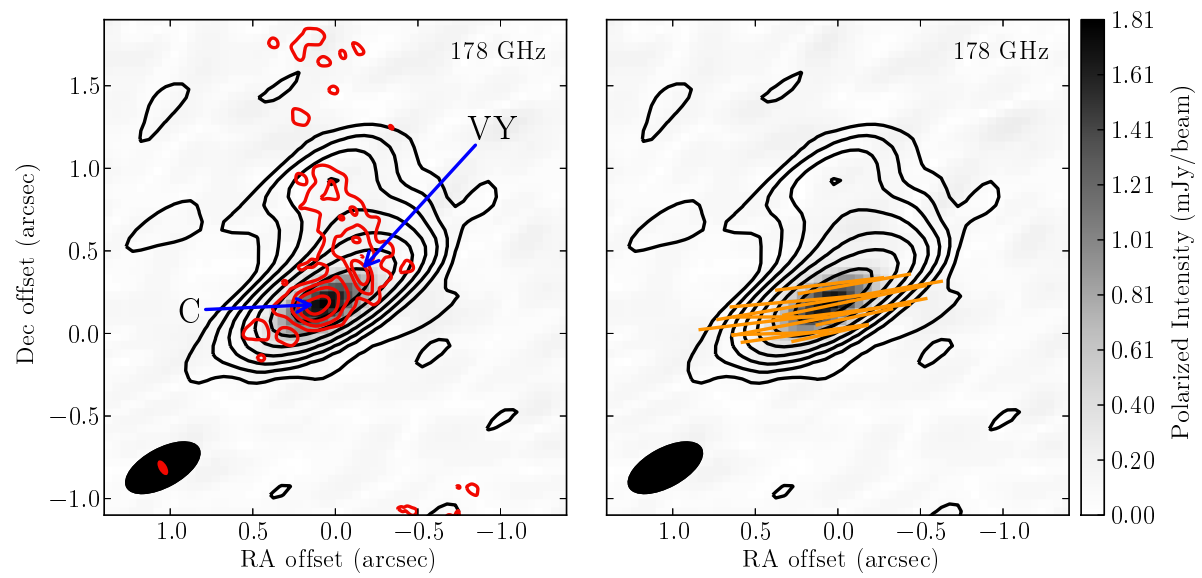

Figure 4. ALMA observations of the dust around the RSG VY CMa at $178 \mathrm{GHz}$ (Vlemmings et al. 2017). Arrows indicate a strong dust clump (C) and the star (VY). The grey scale image is the linearly polarized intensity. The similarly spaced red contours (left) indicate the ALMA $658 \mathrm{GHz}$ continuum from O'Gorman et al. 2015. The vectors (right) indicate the direction of the magnetic field traced by magnetically aligned dust grains.

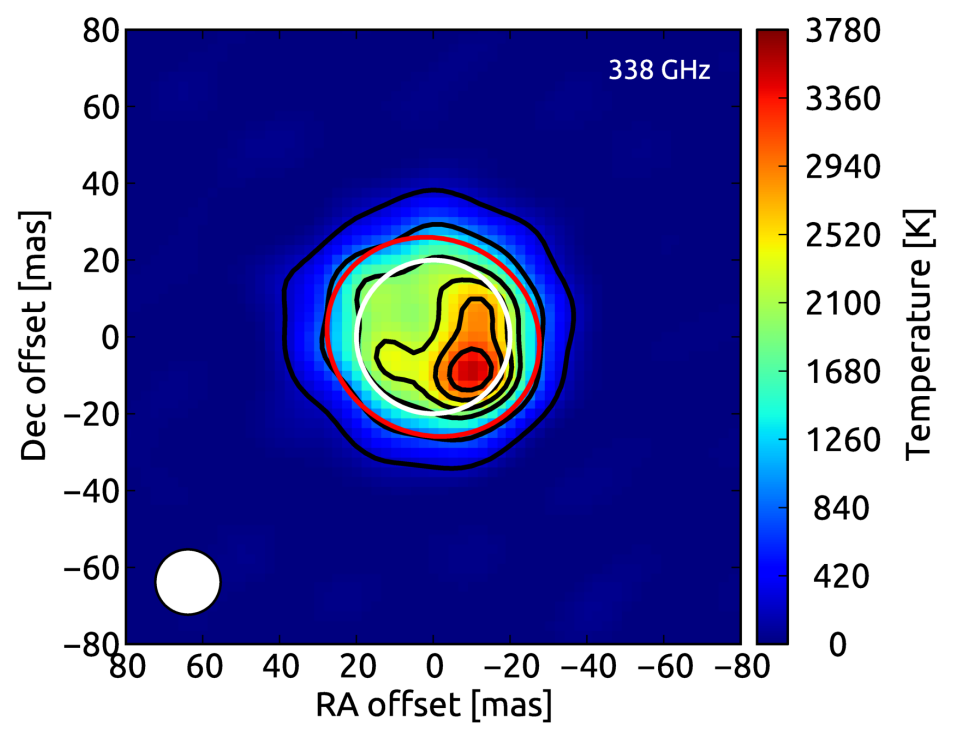

Figure 5. Brightness temperature map of the AGB star W Hya observed with ALMA at 338 $\mathrm{GHz}$ (Vlemmings et al. 2017). The red ellipse indicates the size of the stellar disk at $338 \mathrm{GHz}$ while the white circles indicates the size of the optical photosphere. The clear hotspot is unresolved and it brightness temperature in the map is a lower limit. From size measurements we can constrain the true brightness temperature to be $>50.000 \mathrm{~K}$, which could be a sign of shock interaction or magnetic activity. 

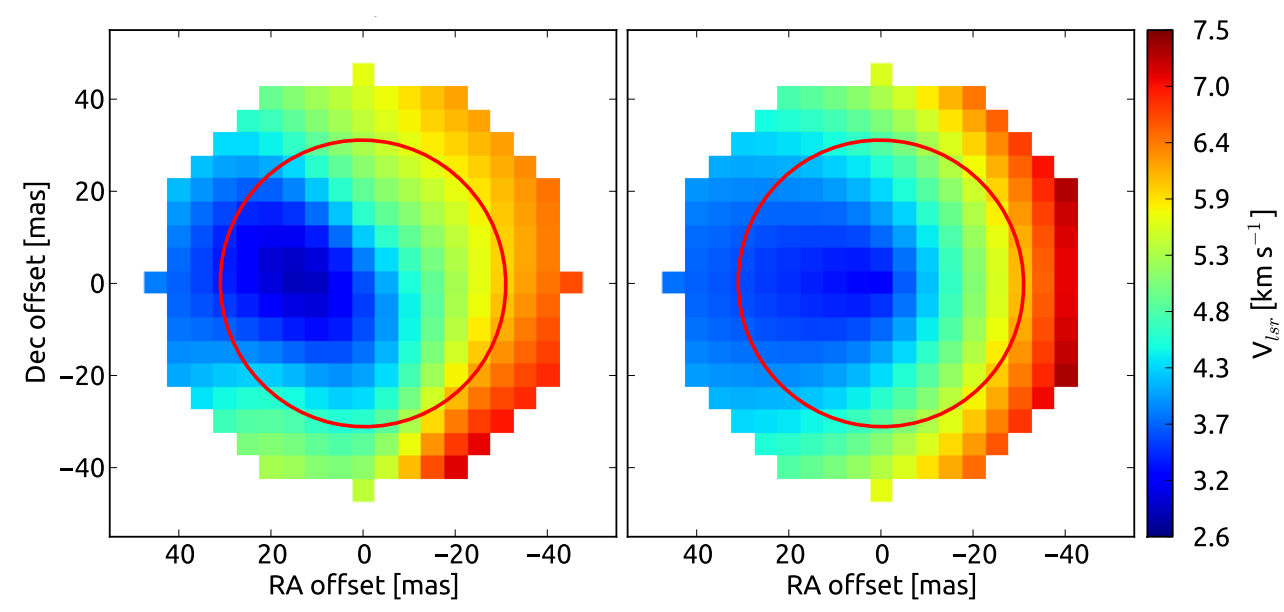

Figure 6. From Vlemmings et al. 2018 (left): Center velocity of the $\mathrm{SiO} v=3, J=5-4$ emission line indicating the fast rotation in the envelope of $\mathrm{R}$ Dot. The red ellipse indicates the measured size of the star at $214 \mathrm{GHz}$. (right): The best fit model of solid-body rotation including a small expansion velocity component.

Vlemmings et al. 2017). These spots can arise from strong shocks but could also point to magnetic activity.

As previously noted, the angular momentum imparted by a stellar (or sub-stellar) companion might be needed to maintain a stellar dynamo that can generate the observed magnetic fields. However, rotation is very difficult to measure for the extended AGB stars that are undergoing pulsations and show large convective cells. Only very recently has ALMA been able to measure the fast $\left(\sim 1 \mathrm{~km} \mathrm{~s}^{-1}\right)$ rotation of the AGB star R Dor (Fig 6 , Vlemmings et al. 2018). As the rotation is almost two orders of magnitude larger than otherwise expected, it is a likely sign of interaction with an hitherto unknown companion. Unfortunately, no magnetic field observations exist yet for $\mathrm{R}$ Dor and it it is thus not yet possible to establish a link between the generation of a magnetic field and the fast rotation.

\section{Conclusions}

Magnetic fields are ubiquitous around AGB and post-AGB stars, and several observations indicate a link between the magnetic field and the collimated outflows found in pre-PNe. Additionally, indirect observations of hotspots and UV-emission might point to magnetic activity on the surface of AGB stars. However, it is only now possible to start probing the morphology of the magnetic field in AGB envelopes and to finally determine the role of magnetism around evolved stars.

\section{References}

Bains, I., Gledhill, T. M., Yates, J. A., \& Richards, A. M. S. 2003, MNRAS, 338, 287

Berdyugina, S. V. 2009, Cosmic Magnetic Fields: From Planets, to Stars and Galaxies, 259, 323

Braithwaite, J., \& Spruit, H. C. 2004, Nature, 431, 819

Charbonneau, P. 2014, ARAA, 52, 251

Duthu, A., Herpin, F., Wiesemeyer, H., et al. 2017, $A \mathscr{E} A$, 604, A12

Falceta-Gonçalves, D. \& Jatenco-Pereira, V. 2002, ApJ, 576, 976

Gómez, J. F., Uscanga, L., Green, J. A., et al. 2016, MNRAS, 461, 3259 
Gonidakis, I., Chapman, J. M., Deacon, R. M., \& Green, A. J. 2014, MNRAS, 443, 3819

Herpin, F., Baudry, A., Thum, C., Morris, D., \& Wiesemeyer, H. 2006, A\&A, 450, 667

Kemball, A. J., Diamond, P. J., Gonidakis, I., et al. 2009, ApJ, 698, 1721

Kwok, S., Purton, C. R., \& Fitzgerald, P. M. 1978, ApJL, 219, L125

Leal-Ferreira, M. L., Vlemmings, W. H. T., Kemball, A., \& Amiri, N. 2013, A\&6A, 554, A134

Lèbre, A., Aurière, M., Fabas, N., et al. 2014, $A \& 4 A, 561$, A85

Montez, R., Jr., Ramstedt, S., Kastner, J. H., Vlemmings, W., \& Sanchez, E. 2017, ApJ, 841, 33

Nordhaus, J., Blackman, E. G., \& Frank, A. 2007, MNRAS, 376, 599

O'Gorman, E., Vlemmings, W., Richards, A. M. S., et al. 2015, A\&A, 573, L1

Pérez-Sánchez, A. F., Vlemmings, W. H. T., Tafoya, D., \& Chapman, J. M. 2013, MNRAS, 436, L79

Rudnitski, G. M., Pashchenko, M. I., \& Colom, P. 2010, Astronomy Reports, 54, 400

Sabin, L., Hull, C. L. H., Plambeck, R. L., et al. 2015a, MNRAS, 449, 2368

Sabin, L., Wade, G. A., \& Lèbre, A. 2015b, MNRAS, 446, 1988

Soker, N. 1998, MNRAS, 299, 1242

Soker, N. 2002, MNRAS, 336, 826

Suárez, O., Gómez, J. F., Bendjoya, P., et al. 2015, ApJ, 806, 105

Thirumalai, A., \& Heyl, J. S. 2013, MNRAS, 430, 1359

Trippella, O., Busso, M., Palmerini, S., Maiorca, E., \& Nucci, M. C. 2016, ApJ, 818, 125

Vlemmings, W. H. T. 2012, in IAU Symposium, Vol. 287, Cosmic Masers - from OH to H0, ed. R. S. Booth, W. H. T. Vlemmings, \& E. M. L. Humphreys, 31-40

Vlemmings, W. H. T. 2014, in IAU Symposium, Vol. 302, Magnetic Fields throughout Stellar Evolution, ed. P. Petit, M. Jardine, \& H. C. Spruit, 389-397

Vlemmings, W. H. T. 2018, Contributions of the Astronomical Observatory Skalnate Pleso, 48, 187

Vlemmings, W. H. T., Diamond, P. J., \& Imai, H. 2006, Nature, 440, 58

Vlemmings, W. H. T., Diamond, P. J., \& van Langevelde, H. J. 2002, A\&A, 394, 589

Vlemmings, W. H. T., Humphreys, E. M. L., \& Franco-Hernández, R. 2011, ApJ, 728, 149

Vlemmings, W. H. T., Khouri, T., De Beck, E., et al. 2018, A\&A, 613, L4

Vlemmings, W. H. T., Khouri, T., Martí-Vidal, I., et al. 2017b, A\&A, 603, A92

Vlemmings, W., Khouri, T., O'Gorman, E., et al. 2017a, Nature Astronomy, 1, 848

Vlemmings, W. H. T., Ramstedt, S., Rao, R., \& Maercker, M. 2012, Aध̈A, 540, L3

Vlemmings, W. H. T., van Langevelde, H. J., \& Diamond, P. J. 2005, A\&A, 434, 1029

\section{Discussion}

De MARCo: There seem to be too many AGB with B-fields to be justified by a close-by companion. So, are you saying that there must be an alternative scenario to the binary scenario?

Vlemmings: Yes. Although the sample can still be considered small, magnetic fields appear to be present in all studies sources with extrapolated surface field strength of a few Gauss. Certainly these sources do not all have close-by stellar companions. 